\title{
PROCESOS DE IDENTIFICACIÓN LITERARIA, UNA VENTANA ABIERTA A LOS UNIVERSOS LITERARIOS ADOLESCENTES: ACERCAMIENTO DESDE UNA PERSPECTIVA DE GÉNERO
}

Marta NÁJERA ARCHILLA

mnarchilla@gmail.com

\section{Resumen}

En este artículo se presentan los resultados de la investigación realizada en el curso 2017-2018 en el I.E.S Medina Albaida (Zaragoza) en torno a los procesos de identificación literaria entre lectores adolescentes, desde una perspectiva de género. Se analizan las respuestas dadas por el alumnado a las cuestiones de la encuesta planteada: ¿Con qué personajes te identificas más, con los masculinos o con los femeninos?, ¿Qué personaje te gustaría ser de los/las que protagonizan los libros que has leído? Asimismo, se presentan, como resultados de la investigación, el pensamiento adolescente, sus universos literarios, estereotipos y roles de género tradicionales enraizados en el pensamiento colectivo. Paralelamente, las conclusiones obtenidas permitirían diseñar propuestas didácticas para prevenir comportamientos sexistas y discriminatorios y tratar de erradicar las violencias de género.

Palabras clave: literatura infantil y juvenil, procesos de identificación literaria, perspectiva de género, adolescencia, roles y estereotipos de género 
Marta Nájera Archilla

\section{LES PROCESUS D'IDENTIFICATION LITTÉRAIRE, UNE FENÊTRE OUVERTE SUR LES UNIVERS LITTÉRAIRES DES ADOLESCENTS: APPROCHE DANS UNE PERSPECTIVE DE GENRE}

\section{Résumé}

Cet article présente les résultats de la recherche réalisée pendant l'année scolaire 2017-2018 à l'Institut d'Education Secondaire Medina Albaida (collège-lycée), à Saragosse sur les processus d'identification 1 ittéraire $\mathrm{d} u \mathrm{l}$ ectorat a dolescent, $\mathrm{d}$ epuis u ne p erspective $\mathrm{d}$ e g enre. S ont analysées les réponses données par les élèves aux questions de l'enquête proposée : Avec quels personnages t’identifies-tu le plus, avec les masculins ou avec les féminins? Quel personnage aimerais-tu être parmi les principaux/principales dans les livres que t'as lus? Sont également inclues, comme des résultats de l'investigation, la pensée des adolescents leurs justifications et leurs univers littéraires adolescents, les stéréotypes et rôles traditionnels de genre enracinés dans la pensée collective. Parallèlement, les conclusions obtenues dans l'étude permettraient de formuler des propositions didactiques pour prévenir des comportements sexistes et discriminatoires et essayer d'éradiquer les violences de genre.

Mots clés : littérature de jeunesse, les processus d'identification littéraire, perspective de genre, adolescence, rôles et stéréotypes de genre

\section{LITERARY IDENTIFIATION PROCESSES, AN OPENED WINDOW TO LITERARY UNIVERSES OF ADOLESCENTS: AN APPROACH FROM A GENDER PERSPECTIVE}

\section{Abstract}

This paper outlines the results of the investigation carried out during the academic year 2017-2018 at Medina Albaida's High School (Saragossa). This research, designed from a gender perspective, focuses on literary identification processes among teenagers readers. The study analyses the answers given by the adolescents to the questions asked on the survey, such as Which characters do you identify 
much more easily with, masculine's or feminine's?, Which character or characters from the books you have read would you like to become? In the same way, teenage thinking and their literary universes, which show traditional stereotypes and gender roles, are presented as findings of this investigation. Meanwhile, the conclusions reached would allow us to design didactic proposals in order to prevent sexist and discriminatory behaviours and eradicate all kind of gender violence.

Key words: children's and young people's literature, literary identification processes, gender perspective, adolescence, gender roles and stereotypes

\section{Introducción}

Este estudio toma como punto de partida la estrecha relación entre la lectura y los procesos de identificación literaria, en c oncreto, e ntre lectores a dolescentes. P or t anto, s e plantea su a nálisis y revisión desde una perspectiva de género tomando como base teórica distintas aportaciones de Silvia Tubert (2008), Antonio Gómez Yebra (2016), Fryda Schulz de Mantovani (1974), Ana Maria Machado (1998), Eloy Martos y Gloria García (2005), Roland Barthes (2007), Teresa Colomer (1997), Consol Aguilar (2007), Seth Lerer (2009) y Victoria Reyzábal (2012). Todo ello, con el propósito principal de constatar en la realidad del marco educativo los procesos de identificación en la adolescencia mediante la Literatura Infantil y Juvenil desde una perspectiva de género.

Para la consecución de tal objetivo, se ha desarrollado una metodología mixta de investigación que ha entrelazado, desde un posicionamiento ecléctico, técnicas y estrategias tanto cualitativas como cuantitativas de recogida y de análisis de datos así como de discusión de resultados. De acuerdo con la población sujeto de la investigación, ésta se ha realizado en el I.E.S Medina Albaida de Zaragoza. Para su desarrollo se ha encuestado al alumnado de los primeros cursos de Educación Secundaria Obligatoria.

Respecto al desarrollo de la investigación, se presentan los datos extraídos y el análisis, desde una perspectiva de género, de las respuestas dadas por el alumnado a determinadas cuestiones de la encuesta como: ¿Con qué personajes te identificas más, con los masculinos o con los femeninos?, ¿Por qué?, ¿Qué personaje te gustaría ser de los/las que protagonizan los libros que has leído?, ¿Por 


\section{Marta Nájera Archilla}

qué? Esta discusión de resultados se ha presentado a través de un enfoque narrativo. Finalmente, se presentan algunas de las conclusiones a las que se ha llegado y que pueden resultar útiles para futuros estudios e incluso para el diseño de propuestas educativas futuras destinadas a prevenir y erradicar las violencias de género.

\section{Los procesos de identificación a través de la Literatura Infantil y Juvenil}

Al enmarcar el estudio a nivel teórico, resulta primordial ofrecer la relación existente entre lectores adolescentes y los procesos de identificación desde una perspectiva de género. Para mostrar la importancia de la lectura y de la literatura en la construcción de sus identidades de género, Silvia Tubert subraya:

[...] el valor de la literatura en este momento de la vida, además de revelar las posibilidades del lenguaje, los libros permiten a las y los adolescentes reconocer y nombrar sus sentimientos y deseos a través de la confrontación con los diversos personajes e identificarse con nuevos modelos y emblemas extra-familiares que pueden enriquecer su ideal del yo (cit. en Martínez Belloch et al., 2008, p. 62).

Generalmente, a lo largo de la etapa de la adolescencia, las y los jóvenes, están pendientes de sí mismos, tanto corporal como psíquicamente; puesto que es un período en el que se enfrentan a nuevas situaciones, en muchas ocasiones problemáticas, todo ello combinado con grandes miedos, inseguridades, pasiones y deseos. Ciertamente, esa amalgama anterior les predispone, como destaca Gómez Yebra (2016), para la lectura de obras encarnadas por personajes con los que puedan identificarse con sus propios sentimientos, conflictos psicológicos, evolución de la personalidad y de la concepción del mundo. De acuerdo con las aportaciones de Aguilar (2007) es necesaria la visibilización del concepto de género que ofrece la LIJ, como instrumento de indagación cultural y como un elemento relevante en la formación de identidades. En definitiva, se destaca esta doble importante función que la LIJ cumple, o puede cumplir, como un elemento de placer y como elemento transformador de desigualdades sociales.

Aun con todo, las obras están destinadas en muchas ocasiones a un rango de edad determinada, debido a lo mencionado anteriormente. No obstante, como advierte Fryda Schulz de Mantovani (1974) "las edades para las que se recomiendan los libros son tan elásticas como el desarrollo mental y emocional de los lectores" (pp. 145-146). En este sentido apuntan Martos y García (2005) para quienes es esencial hacer de las y los lectores jóvenes y de su acompañante, el profesorado, el eje de 
las políticas de la lectura.

Debido al proceso de identificación literaria que tiene lugar, se hace más que necesario revisar las publicaciones que se están realizando, lo cual permite observar el grado de aceptación de determinadas obras, la presencia o no de determinados personajes masculinos y femeninos, los roles que les acompañan, la ideología y los estereotipos presentes, ya que como señala Barthes (2007) hay una identidad entre ideología y estereotipo. Para Teresa Colomer, la LIJ sí que posee una carga ideológica:

$[\ldots]$ que los libros infantiles enseñan a los niños y niñas las convenciones mediante las que su/nuestra cultura acostumbra a contar sus historias literarias. En lo que respecta al itinerario lector resalta que incluso en la posición inicial, los niños y las niñas que leen estos cuentos, ya tienen muchos conocimientos narrativos específicamente literarios que han recibido a través de la explicación oral de cuentos, de los libros para no lectores-as y de los medios audiovisuales (1997, p. 203).

En la misma línea, Ana Maria Machado (1998) sostiene la necesidad de crear textos que "rechacen el estereotipo como punto de partida, que sean distintos, nuevos, únicos en su diferencia y originalidad" (1998, p.62), puesto que según esta autora es lo que les posibilitará “enfrentar la carga de repeticiones, estereotipos y códigos culturales que los atraviesan, a pesar de todo, y ésta es su paradoja y aquí está su fuerza" (1998, p.63).

En relación a la variedad temática, Gómez Yebra (2016) concluye que en la literatura juvenil actual tienen cabida todos los asuntos de la vida cotidiana así como los temas de la vida política y social de diferentes países, todo ello, sin desplazar el interés por los mundos fantásticos y los personajes sobrenaturales. Aun con toda la gran variedad y cantidad de obras escritas y pensadas para un público juvenil, se puede observar que una parte importante de ellas incluye o plantea situaciones cotidianas más o menos problemáticas que deben resolverse. Simultáneamente, el tema del amor aparece en incontables obras destinadas a este público, que se muestra en forma de amistad o como primer amor muy deseado, el mismo que a veces genera sufrimiento, dolor y rencor cuando no llega como se desea; o también, un primer amor que sorprende a los propios protagonistas. De acuerdo con Gómez Yebra (2016), los libros más aceptados por las y los lectores de entre doce y dieciséis años, son primordialmente, aquellos de aventuras cuyos protagonistas son adolescentes de sus mismas edades intentando resolver los problemas que tienen. Estas historias se apoyan en tres ejes esenciales, intriga, misterios y una acción desenfrenada, son las tres claves que atraen a gran parte del público juvenil.

Desde una perspectiva crítica de género, interesa poner el punto de mira en cómo son esas representaciones de las y los personajes de esas obras para poder comprender esos procesos de 


\section{Marta Nájera Archilla}

identificación y entender si existen, o no, estereotipos de género y si se están transmitiendo. Asimismo, interesa ahondar en las justificaciones y explicaciones dadas a dichos procesos de identificación literaria entre adolescentes. En concreto, se problematiza la cantidad y calidad de las representaciones femeninas dentro de un canon literario que para Reyzábal (2012) resulta discriminatorio, puesto que genera desigualdad. En esta línea, Lerer (2009) sostiene que "durante muchísimo tiempo no fue literatura lo efímero, lo popular, lo femenino, lo infantil. La historia de la literatura de las distintas naciones solían ignorar a las mujeres que escribían” (p.17). De hecho, esa infravaloración de la literatura escrita por mujeres está estrechamente relacionada con las representaciones femeninas en la literatura y en concreto, con su falta de representación y los peligros que eso conlleva. En consecuencia, y tomando prestadas las palabras de Scott Momaday, "lo peor que puede sucedernos es que no haya representaciones de nosotros" (cit. en Reyzábal, 2012, p. 22).

\section{Diseño de Investigación: Objetivo, metodología, justificación y c ontextualización d e la}

\section{muestra}

Esta investigación tiene como propósito principal constatar en la realidad del marco educativo los procesos de identificación en la adolescencia mediante la Literatura Infantil y Juvenil desde una perspectiva de género. Para lograr tal objetivo se ha empleado una metodología mixta que ha combinado un enfoque cualitativo mediante la realización de entrevistas al profesorado del centro y distintas técnicas cualitativas de análisis y tratamiento de la información y los datos. A raíz de las cuales se ha optado por la introducción de un enfoque cuantitativo para la recogida de una mayor cantidad de información. En este sentido, tras las entrevistas y su análisis se ha diseñado la herramienta cuantitativa de la encuesta, conformada por 12 preguntas descriptivas y explicativas, combinando algunas de carácter prospectivo y retrospectivo. A lo largo del estudio ambos enfoques se armonizan siguiendo las aportaciones de Hernández Sampieri y Mendoza (2008), "las premisas de ambos paradigmas pueden ser anidadas o entrelazadas y combinadas con teorías sustantivas; por lo cual no solamente se pueden integrar los métodos cuantitativos y cualitativos, sino que es deseable hacerlo" (p. 1). En este sentido, se analizan los datos recogidos desde ambas perspectivas y se emplea un enfoque narrativo para la discusión de los resultados.

En lo relativo a la población, se ha optado por el alumnado de $1^{\circ}$ y $2^{\circ}$ de la E.S.O (Educación 
Secundaria Obligatoria) del I.E.S Medina Albaida siendo la muestra final de un total de 128 adolescentes; 66 alumnas y 62 alumnos de entre 12 y 14 años de edad. Antes del desarrollo de las encuestas, se ha llevado a cabo la validación del instrumento, que se ha realizado mediante el diálogo con profesores, profesoras y a través de su realización con un grupo control de diez alumnas y alumnos de $1^{\mathrm{o}}$ y $2^{\circ}$ E.S.O y una profesora. Respecto a la contextualización del centro, hay que destacar que está situado en el Distrito 8 de Zaragoza ubicado en la zona centro y que acoge a población de clase media y media alta y con alrededor de un $18 \%$ de alumnado de otras nacionalidades.

\section{Los procesos de identificación en la adolescencia mediante la Literatura Infantil y Juvenil}

Este estudio se enmarca dentro un trabajo de investigación más extenso que analiza las respuestas dadas por el alumnado descrito con anterioridad a las doce preguntas de la encuesta diseñada. En concreto el estudio que aquí se desarrolla se centra en las respuestas dadas a las dos cuestiones que conforman la pregunta doce de dicha encuesta. En este sentido, el presente estudio pretende probar si se dan procesos de identificación en la adolescencia a través de la Literatura Infantil y Juvenil y en caso de que así sea, se desea conocer qué modelos y/o referentes literarios/as tiene la adolescencia para identificarse..

En primer lugar, se parte del análisis cuantitativo de las respuestas dadas a la primera parte de la pregunta doce, ¿Con qué personajes te identificas más, con los masculinos o con los femeninos? que de una manera directa interpela a los y las adolescentes a contestar y a reconocer ese proceso de identificación. Hay que puntualizar que la redacción de esta pregunta parte de la premisa de que es más sencillo reconocer si hay o no una identificación planteando una elección con dos opciones explícitas que planteándola de una manera menos concreta. Por consiguiente, para el tratamiento de los datos, se entiende por persona que no reconoce o afirma desarrollar un proceso de identificación con las y los personajes literarios sólo a aquella que responde con términos como ninguno, nadie, no sé o cuando directamente no contesta.

En base a lo anterior, tras el análisis de los datos y tal y como se refleja en el gráfico 1, tan sólo alrededor de un 5\% del alumnado encuestado dice no sentirse identificado con ninguno, pues responde no sé o directamente no contesta. Mientras que el 95\% restante afirma sentirse identificado. 


\section{Marta Nájera Archilla}

\section{Gráfico 1}

Identificación del alumnado con personajes de las obras de LIJ



Fuente: Elaboración propia

No obstante, las contestaciones son variadas, por lo que resulta interesante prestar atención a las respuestas dadas por ese porcentaje alto de alumnado que sí admite cierto proceso de identificación literaria. Conviene recalcar que tras la relectura y análisis de las respuestas ofrecidas se plantean seis categorías para englobar dichas respuestas acerca de con quién se identifican más y son las siguientes; personajes femeninos, masculinos, ambos, ninguno, otros y NS/NC. Todo ello se recoge en el gráfico 2, donde se exponen las respuestas del alumnado, desagregado por género y por curso.

\section{Gráfico 2}

¿Con qué personajes te identificas más, con los masculinos o con los femeninos?

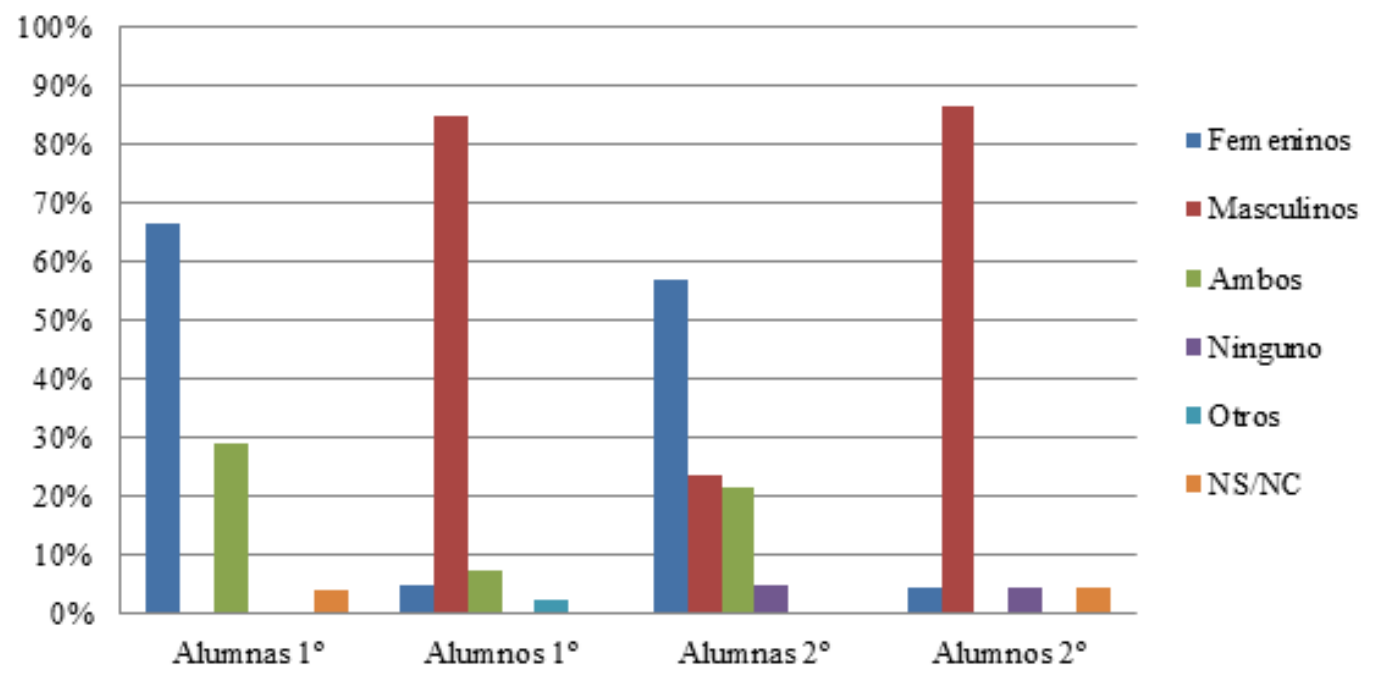

Fuente: Elaboración propia 
En rasgos generales, se observa una mayor identificación con personajes del mismo género que el de las y los lectores. Este aspecto se observa tanto en las como en los adolescentes encuestados. Sin embargo, no se da en la misma medida, según se observa en el caso de $1^{\circ}$ curso; ya que alrededor de un $66,67 \%$ de alumnas se identifican con personajes femeninos frente a un $85 \%$ de alumnos que se identifican con personajes masculinos. Esto muestra que el alumnado femenino de $1^{\circ}$ E.S.O tiene procesos de identificación menos constreñidos a su género, son menos estáticos que el de los alumnos. Lo mismo sucede en el curso siguiente, de hecho, incluso hay una mayor diferencia entre alumnas y alumnos, dado que un $57,14 \%$ de las lectoras dice identificarse con personajes femeninos, mientras que en el caso del público masculino que se identifica con personajes masculinos las cifras alcanzan un $86,36 \%$.

No obstante, estos datos hay que mirarlos con detalle, dado que en cada caso hay una unicidad especial, sobre todo en los motivos ofrecidos para justificar sus respuestas. Por ello, se opta por un análisis cualitativo ${ }^{1}$. Precisamente, estas justificaciones, permiten vislumbrar las diferencias existentes entre las y los protagonistas, si aparece o no una diferenciación sexual, en cuanto a roles, estereotipos y/o mandatos de género para caracterizar a dichos personajes. Como se aprecia en los testimonios siguientes, hay una clara diferenciación entre las conductas, actitudes y roles que adoptan las y los protagonistas de las obras leídas. En esta dirección apuntan las alumnas 1 y 50, al afirmar que los personajes masculinos y femeninos piensan de distinta forma.

- Alumna 1, (13 años): Con los femeninos. Porque los masculinos y femeninos piensan de distinta forma

- Alumna 50, (14 años): Con los femeninos porque yo soy una chica y pues las chicas tenemos una manera distinta de pensar a los chicos.

En otros casos, se sostiene esa idea por la facilidad de identificación con los personajes, bien sea porque se comparten preferencias y/o sentimientos.

1 Nota: Se ha mantenido la escritura y ortografía original de las respuestas dadas por el alumnado siendo fiel a los textos originales 


\section{Marta Nájera Archilla}

- Alumna 3, (12 años): Con los femeninos. Porque la mayoría de personajes femeninos tienen los mismos gustos que yo

- Alumna 15, (- años): Con los femeninos porque puedo saber más fácilmente que es lo que sienten

- Alumna 54, (13 años): Con los femeninos porque hablan sobre chicas y lo incómodo que es hablar con chicos.

- Alumna 2, (12 años): Con los femeninos. Porque me puedo imaginar que soy yo la protagonista y meterme en el papel

Sin embargo, resulta llamativa la afirmación de la alumna 16, quien sostiene que los personajes femeninos tienen más sentimientos y que eso es precisamente lo que facilita su identificación.

\section{- Alumna 16, (13 años): Con los femeninos porque tenían más sentimientos}

Paralelamente, tal y como muestran los extractos de las alumnas 17, 28 y 29, también se habla de discriminación, de roles sexuados diferenciados e incluso de la ausencia de mujeres en los libros de historia.

- Alumna 17, (13 años): Con los femeninos. Me parece que los personajes femeninos pueden ser fuertes e independientes o no y ese cambio me parece necesario para variar los libros

- Alumna 28, (14 años): Con los femeninos. Porque estamos demasiado discriminadas y creo que ahora muchos libros en vez de ser personajes masculinos deberían ser femeninos y hacernos protagonistas absolutas

- Alumna 29, (14 años): Con los femeninos. Porque además de ser mujer, cuando leo libros de historia sobre todo me enfurece saber que pocas mujeres aparecen en ellos además de que muchas mujeres han cambiado nuestra historia

Algunos de los aspectos destacados aparecen en la mayoría de las respuestas dadas por las alumnas y se contraponen a gran parte de las de los alumnos. Como se muestra con los ejemplos siguientes, muchos de los adolescentes reducen su identificación por el género al que pertenecen, de modo 
que como refleja el alumno 13, para algunos de ellos es impensable el poder identificarse con otros personajes que no sean los masculinos.

- Alumno 13, (12 años): Con los masculinos, porque soy hombre?

Para otros como el alumno 11, el criterio tiene más que ver con la personalidad y con el carácter que poseen estos personajes, de lo que se deduce que es ampliamente diferente del resto de personajes.

- Alumno 11, (14 años): Con los masculinos, por el carácter que adoptan

- Alumno 21, (15 años): Con los masculinos, por que algunos cometen los mismos errores que cometo yo

- Alumno 24, (13 años): Con los masculinos. Los entiendo más que a los femeninos

Algunos como el alumno 12, hacen hincapié en los comportamientos y en las actividades que realizan; puesto que las considera similares a las que él desempeña.

- Alumno 12, (13 años): Con los masculinos. Porque en este caso los personajes masculinos tienen más relación con las acciones que yo realizo

Otros, como el alumno 27, ponen de relieve una cuestión importante, la lectura de libros con protagonistas del mismo género que el lector, que en muchas ocasiones dificulta la posible identificación con otras identidades, así como la asimilación del protagonismo masculino como algo incuestionable.

- Alumno 27, (13 años): Con los masculinos porque solo e leído libros con protagonistas masculinos

Otro de los aspectos clave para observar esta mayor rigidez y estatismo del alumnado masculino, se vislumbra si se presta atención a la categoría ambos. Desde un análisis cuantitativo, esta respuesta ha sido dada por un $29,17 \%$ de alumnas de primer curso frente a un 7,5\% de alumnos, las discrepancias aún son mayores en segundo curso, ya que el 21,43\% del público femenino de ese curso dice sentirse identificada con personajes masculinos y femeninos mientras que ningún alumno del mismo curso 


\section{Marta Nájera Archilla}

responde lo mismo. En relación a lo anterior, se ha recogido el testimonio de aquellas personas que afirman sentirse identificadas con ambos personajes. Tras la revisión de las encuestas, se ha observado que en los casos en los que se sienten identificados con ambos personajes son mayoritariamente las adolescentes y no los adolescentes. Ante las limitaciones del análisis cuantitativo se opta por introducir un análisis cualitativo. Por ello, se han incluido algunos testimonios que ellas han ofrecido. Muchas de las alumnas dicen no prestar importancia a si es masculino o femenino, puesto que hay otras cosas más relevantes como apuntan las alumnas 81 y 83 .

- Alumna 81, (13 años): Con ambos según que libros. En todos los libros que he leído me identifico con un personaje sin importar si es chico o chica

- Alumna 83, (12 años): Los dos. En los personajes no importa el sexo sino lo que siente y cómo es

Para otras, como las alumnas 14 y 15, tiene que ver con las actividades y con la toma de iniciativa que realizan los personajes masculinos y con los sentimientos y el aspecto en relación a los femeninos. Lo mismo sucede con las alumnas 82 y 31, para ellas se debe darse una combinación de ambas características para sentirse definidas porque no encajan en el patrón de feminidad hegemónico y por lo tanto comparten acciones y actividades tradicionalmente asociadas a la masculinidad hegemónica.

- Alumna 15, (12 años): Con los dos porque una parte los masculinos tienen sus sentimientos como las mujeres casi todos somos iguales

- Alumna 14, (13 años): Con los dos. Porque en algunos casos llego a entender más al otro género, pero más bien depende de los pensamientos de ese personaje, no sobre si es chico o chica

- Alumna 82, (13 años): Depende, a veces con unos y otras veces son otros. Porque creo que siempre los personajes que se identifican conmigo, que son los deportistas, los que llevan la iniciativa y los más alegres son los masculinos, pero a veces cuando los describen de aspecto me parezco más a los femeninos 
- Alumna 31, (13 años): Depende de como sea el personaje. Si el personaje, ya sea chico o chica, se parece a mí, pues me identifico con él/ella

No obstante, también hay alumnos, aunque en menor medida, que se identifican con ambos personajes como subrayan los alumnos 88,89 y 90 .

- Alumno 88, (13 años): Con ambos porque aveces depende como sea el papel

- Alumno 89, (13 años): Con ambos porque podría estar de cada tipo, y cada personaje en cada historia tiene su propia importancia

- Alumno 90, (12 años): Con ambos porque a mí me suele pasar lo mismo que a ellos

El último de los elementos más relevantes del gráfico 2 es lo que respecta al alumnado que se identifica con personajes distintos a su género. Cabe subrayar que en el caso de los alumnos, son tres los que dicen identificarse con personajes femeninos. Debido al reducido número que representan, se considera oportuno incluir las explicaciones ofrecidas con intención de profundizar en su argumentario.

- Alumno 9, (13 años): Con los femeninos. Porque en general esos personajes suelen esconder mucho poder y acaban siendo más poderosos que los masculinos

- Alumno 10, (12 años): Con los femeninos. Porque una personaje tenía un carácter parecido al mío

- Alumno 26, (13 años): Con los femeninos porque son más ordenados y tienen paciencia

Estos casos, ofrecen una perspectiva transgresora, en tanto que salen de los tradicionales estereotipos y roles de género, permitiendo romper estos planteamientos tan estáticos, cerrados y exclusivos. Al mismo tiempo, nos ofrecen otras ideas asociadas a los personajes femeninos, como el poder que en tantas ocasiones monopolizaban los personajes masculinos. El último, pone en valor algunos de los estereotipos asociados a la feminidad como el orden y la paciencia, con los que reconoce sentirse identificado. 


\section{Marta Nájera Archilla}

Curiosamente, son muchas más las transgresiones que se dan entre el alumnado femenino, habiendo una mayor cantidad de alumnas que dicen identificarse con personajes masculinos. Como se aprecia en los ejemplos recogidos a continuación, los motivos son variados, desde el interés o aficiones comunes según las alumnas 30 y 32 .

- Alumna 30, (13 años): Con los masculinos. Porque son casi como yo, mis gustos...

- Alumna 32, (13 años): Con los masculinos, porque hablan del campo y de la caza y a mí me gusta aunque sea chica

Para otras alumnas, este proceso de identificación resulta del protagonismo y del papel activo que desempeñan estos personajes, de las capacidades de resolver, del éxito e incluso de la heroicidad atribuida a dichas actuaciones.

- Alumna 33, (14 años): A veces con los masculinos porque normalmente en los libros ellos suelen ser los héroes que solucionan todo

- Alumna 34, (14 años): Con los masculinos porque se esfuerzan para conseguir las cosas

- Alumna 35, (13 años): Con los masculinos porque son valientes y decididos

De forma similar, resulta llamativo que nadie haya hecho referencia a otras identidades que no sean las del sistema hegemónico binario masculino-femenino. Por ello, y para ahondar de forma más específica, se desea conocer con qué figuras o personajes concretos, de las obras que han leído y que leen, les gustaría ser y las razones que alegan a dicha decisión. Ante lo cual, se considera relevante la segunda parte de esta pregunta doce en donde se pregunta al alumnado ¿Qué personaje te gustaría ser de los/las que protagonizan los libros que has leído? ¿Por qué? Se podría pensar, como hipótesis inicial que muchas de las respuestas dadas irán en relación a lo que el propio alumnado habría contestado en el apartado anterior, es decir, que los que decían identificarse con personajes masculinos escogerán protagonistas masculinos, y lo mismo sucederá con los personajes femeninos. De hecho, a pesar de la variedad de respuestas recogidas, una amplia mayoría del alumnado encuestado se identifica con su propio género, tal y como se refleja en las tablas a continuación. En donde se exponen aquellos 
Procesos de identificación literaria, una ventana abierta a los universos literarios adolescentes...

personajes que al alumnado de $1^{\circ}$ y $2^{\circ}$ E.S.O le gustaría ser, así como las obras literarias en las que aparecen.

Tabla 1

Personajes y títulos del alumnado masculino de $1^{\circ}$ y $2^{\circ}$ E.S.O

\begin{tabular}{|c|c|}
\hline \multicolumn{2}{|c|}{ ALUMNOS $1^{\circ}, 2^{\circ}$ E.S.O } \\
\hline PERSONAJES MASCULINOS (32) & TÍTULO OBRA \\
\hline $\begin{array}{l}\text { Pakete } \\
\end{array}$ & Los futbolísimos \\
\hline Sam & Los legados de Lorien \\
\hline RomWeasley & Harry Potter \\
\hline Detective Conan & Detective Conan \\
\hline Carter Kane & Hermanos Kane \\
\hline Segismundo & Segismundo y compañia \\
\hline Hamza Zaidi & Kitipasa: Escoge tu propia locura \\
\hline Newt & Themazerunner \\
\hline Protagonista chico & El misterio del Samurai \\
\hline Spiderman & Spiderman \\
\hline Tom Gates & El genial mundo de Tom Gates \\
\hline Kim & Kim \\
\hline Tintin & Las aventuras de Tintin \\
\hline El naúfrago & Diario de un náufrago \\
\hline Elvis Riboldi & Jo, Elvis Riboldi \\
\hline Superman, Spiderman & Superman, Spiderman \\
\hline Goku & DragonBall \\
\hline Vegeta & DragonBall \\
\hline Luh (youtuber) & Tragamundos \\
\hline Arnau Queralt, hacker que se lleva a todas & Los orígenes perdidos \\
\hline Rodrigo Díaz de Vivar & El Cid campeador \\
\hline D'Artagnan & Los tres mosqueteros \\
\hline Magnus Chase & Magnus Chase y los dioses de Asgard \\
\hline IvanFardiño & Ivan Fardiño: mañana seré libre \\
\hline El maestro Hora & Momo \\
\hline Martín de los Narces & La dama del alba \\
\hline Grey & 50 sombras de Grey \\
\hline El protagonista & It \\
\hline El abuelo & La dama del alba \\
\hline Light Yagami (el malo) & Death note \\
\hline Izuku & Boku mi heroacademy \\
\hline
\end{tabular}


Marta Nájera Archilla

\begin{tabular}{|c|c|}
\hline \multicolumn{2}{|c|}{ ALUMNOS $\mathbf{1}^{\mathbf{0}} \mathbf{2}^{\mathbf{0}}$ E.S.O } \\
\hline OTROS PERSONAJES (4) & TÍTULO \\
\hline El loro & Segismundo y compañía \\
\hline El Protagonista & El fútbol es así \\
\hline El bueno, el chico & \\
\hline
\end{tabular}

Fuente: Elaboración propia

Tabla 2

Personajes y títulos del alumnado femenino de $1^{\circ}$ y $2^{\circ}$ E.S.O

\begin{tabular}{|c|c|}
\hline \multicolumn{2}{|c|}{ ALUMNAS $1^{\circ}, 2^{\circ}$ E.S.O } \\
\hline PERSONAJES FEMENINOS (37) & TÍTULO \\
\hline Tris & Divergente \\
\hline MacKenzie & Atrévete a enamorarte \\
\hline Ana Shirley & De casi todo se aprende \\
\hline Katniss & Los juegos del hambre \\
\hline Luna & Mystical: El despertar de la magia \\
\hline Protagonista & $\begin{array}{l}\text { Algo tan sencillo como twittear te quie- } \\
\text { ro }\end{array}$ \\
\hline Agatha Christie & Casos de Poirot \\
\hline Luna Lovegood & Harry Potter y la orden del Fénix \\
\hline La espectadora & Segismundo y compañia \\
\hline Blanca & Pkm Negro y Pkm Blanco \\
\hline La mujer misteriosa & Las almas de Brandon \\
\hline Teresa & El corredor del laberinto \\
\hline La hermana & El club de las zapatillas rojas \\
\hline Hannah & 13 razones \\
\hline Adela & La dama del Alba \\
\hline Angélica & La dama del Alba \\
\hline Mónica Estés & El club de las mujeres mutantes \\
\hline La Reina Isabel la Católica & \\
\hline Cleopatra, Agualuna & Agualuna \\
\hline L. Lovegood & Harry Potter \\
\hline Telva & La dama del alba \\
\hline Cenicienta & Cenicienta \\
\hline Bella (la protagonista) & Crepúsculo \\
\hline Puck & Puck \\
\hline Diana & Crónicas de la torre \\
\hline Lily & Otoño en Londres \\
\hline Amigas de Hannah & 13 razones \\
\hline
\end{tabular}


Procesos de identificación literaria, una ventana abierta a los universos literarios adolescentes...

\begin{tabular}{|c|c|}
\hline Victoria & $\begin{array}{c}\text { Memorias de Idhún } \\
\text { Harry Potter }\end{array}$ \\
\hline Lermione Granger & $\begin{array}{c}\text { He consejos para sobrevivir a tus ami- } \\
\text { gos.. }\end{array}$ \\
\hline Margó & Ciudades de papel \\
\hline La peregrina & La dama del Alba \\
\hline Brenda & The Marre runner PotterThe Maze Runner \\
\hline M. McGonagall,Hanna Abott & De (casi) todo se aprende \\
\hline Paula Gonu & TíTULO \\
\hline OTROS PERSONAJES (6) & $\mathbf{1}^{\mathbf{0}}, \mathbf{2}^{\mathbf{0}}$ E.S.O \\
\hline La más inteligente & \\
\hline Protagonista & \\
\hline Los que resuelven & \\
\hline Los valientes & \\
\hline El bueno & \\
\hline El policía & \\
\hline
\end{tabular}

Fuente: Elaboración propia

A pesar de lo mencionado con anterioridad, hay un número de alumnos y alumnas que contradicen la respuesta dada inicialmente y que reconocen identificarse con personajes de otro género distinto al suyo. Como se muestra en la siguiente tabla:

Tabla 3

Alumnado de $1^{\circ}$ y $2^{\circ}$ E.S.O que se identifica con personajes de otro género distinto al suyo

\begin{tabular}{|c|c|}
\hline \multicolumn{2}{|c|}{ ALUMNOS $\mathbf{1}^{\mathbf{0}} \mathbf{2}^{\mathbf{0}} \mathbf{\text { E.S.O }}$} \\
\hline PERSONAJES MASCULINOS (4) & TÍTULO OBRA \\
\hline La Mujer invisible & Los cuatro fantásticos \\
\hline Cristal & Pkm \\
\hline Irene Adler & Sherlock Holmes \\
\hline Katniss & Los juegos del hambre \\
\hline
\end{tabular}




\section{Marta Nájera Archilla}

\begin{tabular}{|c|c|}
\hline \multicolumn{2}{|c|}{ ALUMNAS $\mathbf{1}^{\mathbf{0}}, \mathbf{2}^{\mathbf{0}}$ E.S.O } \\
\hline PERSONAJES MASCULINOS (12) & TÍTULO \\
\hline Al chico al que se dirige el libro & El príncipe de mis sueños \\
\hline Thomas II & El corredor del laberinto \\
\hline Hugo & Abajo el colegio \\
\hline Kim & Kim \\
\hline H.Potter & Harry Potter \\
\hline D. Malfoy & Harry Potter \\
\hline Poe & El misterio de la calle Morgue \\
\hline Benjamin & El medallón perdido \\
\hline Minho & The Maze runner \\
\hline N. Longbotton, & Harry Potter \\
\hline R. Lupin & The Maze Runner \\
\hline S. Black, & Harry Potter \\
\hline
\end{tabular}

Fuente: Elaboración propia

Hay algún caso de los expuestos en la tabla 3 que desde un análisis cualitativo merece cierta reflexión, empecemos por el alumnado masculino que ocupa la parte superior de la tabla y que son del total de alumnos encuestados, los únicos que afirman identificarse con algún personaje femenino. Precisamente, de estos cuatro únicos casos, dos de ellos, en la primera parte de la pregunta contestaron que se sentían más identificados con los personajes masculinos. Sin embargo, a posteriori, escogieron como personaje que les gustaría ser, uno femenino. Como sucede con el alumno 104 (13 años) que quiere ser Cristal, personaje femenino de Pokémon y la explicación que ofrece es porque vive en el mundo de Pokémon. Igualmente ocurre con el alumno 41(14 años) a quien le gustaría ser Katniss, la protagonista femenina de la saga de Los juegos del hambre, porque según él es una luchadora y nunca se rinde. Aunque estos casos, muestran la posibilidad de transgredir los estereotipos y roles asignados, la realidad es que tan solo un $6,45 \%$ de los alumnos encuestados se identifican o admiten identificarse con uno o más personajes femeninos.

Si se comparan los datos anteriores con el caso de las alumnas se pueden apreciar notables diferencias; ya que hay tres veces más cantidad de alumnas que de alumnos que no solo se identifican con personajes de otro género distinto al suyo sino que les gustaría ser uno de esos personajes, alrededor de un 20\%. Dado el elevado número, resulta relevante revisar y reflexionar acerca de las explicaciones ofrecidas por las alumnas. Por ello, se considera oportuna la inclusión de algunos testimonios. Como el de la alumna 81 (13 años) que le gustaría ser Thomas, el protagonista masculino de la saga El corredor 
del laberinto, porque según ella es valiente y para él los amigos es lo primero. Además de que en el libro está muy bien descrito y parece muy real y a veces me identifico con él. A la alumna 62 (14 años) le gustaría ser Benjamín, protagonista de la obra El medallón perdido porque tiene casi mi edad y vivió aventuras muy chulas. Otras, como las alumnas 33 y 34 (14 años ambas), señalan que les gustaría ser los personajes principales porque son los que viven más aventuras.

\section{Conclusiones}

A raíz de esta investigación, se puede deducir que sí que se dan procesos de identificación literaria, es más, tras analizar las explicaciones y justificaciones del alumnado adolescente, se aprecia que desde una perspectiva de género, estos procesos sí que tienen repercusiones en sus deseos, expectativas, consideraciones acerca de las personalidades, comportamientos, actitudes, valores e identidades más o menos idealizadas. No solo eso, sino que se siguen y prescriben unos estereotipos y roles de género a dichas representaciones y por ende, se mantienen mediante dichos procesos de identificación literaria.

Aunque el argumentario sea variado, parecen confluir muchos de los motivos en el mayor protagonismo, en las actividades y roles que desempeñan esos personajes masculinos, en el modo de relacionarse, en la toma de decisiones, en el carácter aventurero o en las actitudes de liderazgo y de toma de iniciativa lo que resulta interesante y deseoso o incluso como un ideal de personalidad para gran número de alumnas. Quizá, muchas de las lectoras se sientan identificadas con estos personajes masculinos por estas razones, bien porque haya un mayor protagonismo masculino en las obras de literatura juvenil o porque los valores, actitudes, comportamientos y expectativas de los personajes femeninos no sean los que más atraigan al público femenino. Lo que sí que parece claro, fuere la razón que fuere, es que un mayor número de lectoras se identifican con protagonistas masculinos que el número de lectores que lo hacen con personajes femeninos.

A colación de la observación anterior, se exige prestar atención a la dificultad de muchos alumnos para identificarse con personajes femeninos, lo cual puede hacernos pensar en la posibilidad de que se sustenten y/o refuercen la idea asociada al imaginario colectivo tradicional y patriarcal. Según este imaginario heteropatriarcal, tradicionalmente se ha dado una infravaloración de lo femenino o todo lo que se piense asociado a ello. Por ende, la LIJ permite acercar y ofrecer a la adolescencia la oportunidad de conocer a personajes femeninos reales. En consecuencia, se facilitaría y promovería el desarrollo 


\section{Marta Nájera Archilla}

de actitudes como la empatía, el respeto, el reconocimiento y la puesta en valor de lo femenino permitiendo unas mejores relaciones entre las personas en el presente y en el futuro, fomentando los cuidados y la no discriminación como herramientas para prevenir los estereotipos discriminatorios y sexistas. Si no, lo que podría suceder es que se corra el riesgo de caer en la infravaloración de lo otro, por simple desconocimiento, falta de identificación y/o de empatía, provocando que los estereotipos y roles de género sustituyan un pensamiento crítico no discriminatorio.

\section{Referencias bibliográficas}

Aguilar, C. (2007). Del discurso de la domesticidad a la cultura queer en la literatura infantil y juvenil. ¿Todas las mujeres podemos?: Género, Desarrollo y multiculturalidad. III Congreso estatal de la Fundación Isonomía para la igualdad de oportunidades entre mujeres y hombres (pp. 62-69). En línea http://citeseerx.ist.psu.edu/viewdoc/ download?doi=10.1.1.848.3194\&rep=rep1\&type=pdf\#page=63. Castelló: Universitat Jaume I. Última consulta: 26 -1-2019.

Barthes, R. (2007). El placer del texto; y Lección inaugural. Madrid: Siglo XXI de España Editores. Colomer, T. (1997). Cómo enseñan a leer los libros infantiles. In Didáctica de la lengua y la literatura para una sociedad plurilingüe del siglo XXI (pp. 203-208). Barcelona: Universidad de Barcelona. Servicio de Publicaciones.

Gómez Yebra, A. (2016). Animación a la lectura y literatura juvenil. Sevilla: Editorial Renacimiento. Hernández Sampieri, R., \& Mendoza, C. P. (2008).El matrimonio cuantitativo cualitativo: el paradigma mixto. In JL Álvarez Gayou (Presidente), $6^{\circ}$ Congreso de Investigación en Sexología. Congreso efectuado por el Instituto Mexicano de Sexología, AC y la Universidad Juárez Autónoma de Tabasco, Villahermosa. Tabasco, México.

Lerer, S. (2009). La magia de los libros infantiles: de las fábulas de Esopo a las aventuras de Harry Potter. Barcelona: Editorial Crítica. Col. Ares y Mares.

Machado, A. M. (1998). Buenas palabras, malas palabras. Buenos Aires: Ed. Sudamericana.

Martínez Belloch, I., Amigot Leache, P., Bayot Mestre, A., Bonilla Campos, A., Castillo Martín, M., Gómez Sánchez, P.,... \& Mira Pérez, J. (2008). Imaginario cultural, construcción de identidades 
Procesos de identificación literaria, una ventana abierta a los universos literarios adolescentes...

de género y violencia: formación para la igualdad en la adolescencia. Madrid: Instituto de la Mujer.

Martos, E. y García, G. (2005). Las nuevas tecnologías y la literatura infantil y juvenil. In Lectura y literatura infantil y juvenil: claves (pp. 247-284). Málaga: Ediciones Aljibe.

Reyzábal, M. V. (2012). Canon literario y diferencia de género en la educación.Madrid: La Muralla.

Schultz De Mantovani, F. (1974). Sobre las hadas. Buenos Aires: Nova. 